\title{
Secondary Rayleigh-Taylor type Instabilities in the Reconnection Exhaust Jet as a Mechanism for Supra-Arcade Downflows
}

\author{
L.-J. Guo ${ }^{1,2,3,4}$ \\ lijiag@princeton.edu \\ A. Bhattacharjee $e^{1,2,3,4}$ \\ amitava@princeton.edu \\ Y.-M. Huang ${ }^{1,2,3,4}$ \\ yiminh@princeton.edu \\ and \\ D. E. Innes ${ }^{4,5}$ \\ innes@mps . mpg . de
}

Received — $—$ accepted

\footnotetext{
${ }^{1}$ Space Science Center, University of New Hampshire, Durham, NH 03824, U.S.A.

${ }^{2}$ Department of Astrophysical Sciences and Princeton Plasma Physics Laboratory, Princeton University, Princeton, NJ 08540, U.S.A.

${ }^{3}$ Max Planck/Princeton Center for Plasma Physics, Princeton, NJ 08540, U.S.A.

${ }^{4}$ Max-Planck Institute for Solar System Research, Göttingen 37077, Germany
} 


\begin{abstract}
Supra-arcade downflows (hereafter referred to as SADs) are low-emission, elongated, finger-like features usually observed in active-region coronae above post-eruption flare arcades. Observations exhibit downward moving SADs intertwined with bright upward moving spikes. Whereas SADs are dark voids, spikes are brighter, denser structures. Although SADs have been observed for decades, the mechanism of formation of SADs remains an open issue. In our three-dimensional resistive magnetohydrodynamic simulations, we demonstrate that secondary Rayleigh-Taylor type instabilities develop in the downstream region of a reconnecting current sheet. The instability results in the formation of low-density coherent structures that resemble SADs, and high-density structures that appear to be spike-like. Comparison between the simulation results and observations suggests that secondary Rayleigh-Taylor type instabilities in the exhaust of reconnecting current sheets provide a plausible mechanism for observed SADs and spikes.
\end{abstract}

Subject headings: <Sun: flares, instabilities, magnetic reconnection> 


\section{Introduction}

Supra-arcade downflows (hereafter referred to as SADs) (also known as tadpoles due to their wavy appearance) are low-emission, elongated features usually observed in active-region coronae above post-eruption flare arcades (McKenzie \& Hudson 1999 . McKenzie 2000). SADs are usually observed in the extreme-ultraviolet (EUV) and X-ray filter images that detect plasma in the temperature range $10^{6.8}-10^{7.3}$ Kelvin, and they have a typical life time of a few minutes. By using the filter ratio method (Hara et al. 1992) to deduce the temperature with the data from the Soft X-ray Telescope (SXT), McKenzie \& Hudson (1999) showed that SADs are low-density $\left(<10^{9} \mathrm{~cm}^{-3}\right)$, high-temperature $\left(\sim 10^{7} K\right)$ structures. This result is supported by Savage \& McKenzie (2012) as well as the SUMER spectroscopic analysis conducted by Innes et al. (2003a). Asai et al. (2004) found that the occurrences of SADs are highly correlated with nonthermal bursts in microwave and hard X-ray (HXR), suggesting that the formation of SADs involves magnetic reconnection or consequent outflows. Innes et al. (2003b) reported high Doppler-shifted Fe XXI line profiles at the edges of SADs, corresponding to line-of-sight velocity up to 1000 $\mathrm{km} / \mathrm{s}$. However, the source of the observed high-velocity is not clearly established by these

studies. More recently, Savage \& McKenzie (2011) conducted a statistical study and found that the average velocity of most SADs is around $150 \mathrm{~km} / \mathrm{s}$, which is a fraction of the typical Alfvén speed ( $1000 \mathrm{~km} / \mathrm{s})$ of the supra-arcade plasma in corona. Furthermore, McKenzie (2013) performed local correlation tracking (LCT) on sequences of EUV images and found that vortices existed at the regions where SADs were observed.

It is important not to confuse SADs with plasmoids or magnetic islands. Observationally, SADs are density depletion regions (Savage \& McKenzie 2012; Innes et al. 2003a), whereas plasmoids are usually density-enhanced structures (Lin et al. 2005, Liu et al. 2010). Plasmoids are observed edge-on as bright blobs moving along the post-CME current 
sheet, whereas SADs are most clearly visible when observing the current sheet and the underlying arcade face-on. The SADs and spikes are seen intermittently in the direction perpendicular to the underlying arcades and appear to be flute-like (i.e. $\mathbf{k} \cdot \mathbf{B} \simeq 0$, where $\mathbf{k}$ is the wave number and $\mathbf{B}$ is the magnetic field). This distinction is clearly illustrated in Asai et al. (2004) and Savage \& McKenzie (2012).

Although SADs have been the subject of significant theoretical research during the past decade, the physical mechanisms that drive the formation of finger-like SADs and spikes remain under debate. There have been some simulations attempting to reproduce the observational features of SADs in the literature. The "patchy reconnection" model (e.g., Linton \& Longcope 2006, Linton et al. 2009) uses spatially localized anomalous resistivity intermittently over time along the current sheet layer to trigger intermittent reconnection. The reconnected magnetic field lines then cause intermittent disturbances in the current sheet as they contract toward the downstream region, and the flux tubes that emerge, with tear drop-like cross-sections, have been interpreted as SADs. To test this idea, Scott et al. (2013) attempt to reproduce SADs in simulation as the wakes caused by reconnected flux tubes moving at a high speed. On the other hand, the SAD model developed by Costa et al. 2009), Maglione et al. (2011), and Cécere et al. (2012) assumes multiple reconnection sites in which the SADs are a consequence of shocks and rarefactions bouncing back and forth in magnetic structures. In these studies, magnetic reconnection is not directly simulated; instead, reconnection ejections are modeled with localized pressure enhancements in the initial condition. Recently, Cassak et al. (2013) proposed that SADs are flow channels carved by low-density, sunward-directed reconnection jets in high-density underlying arcades. In this scenario, reconnection is continuous in time so that the SADs are not filled in from behind as they would be if they were caused by isolated descending flux tubes; however, reconnection has to be spatially localized to keep the outflow jet collimated. 
The studies mentioned above have a common feature that reconnection has to be at least spatially localized, while in some scenarios reconnection has to be temporally localized as well. In this Letter, we show that the finger-like SADs can arise as a result of secondary Rayleigh-Taylor type instabilities in the downstream region of reconnection in a post-eruption current sheet. This physical mechanism was first suggested in Asai et al. (2004) and explored partially by TanDokoro \& Fujimoto (2005) by means of magnetohydrodynamic (MHD) simulations primarily in the context of the Earth's magnetotail. In this work we carry out two simulations, one with uniform resistivity and the other with spatially localized anomalous resistivity. We find that Rayleigh-Taylor type instabilities arise in both simulations. Dynamic features of the instabilities exhibit good agreement with observations of SADs, and the emulated synthetic emission count rate from simulations also shows qualitative similarities with extreme ultra-violet (EUV) images obtained from the Atmospheric Imaging Assembly (AIA) on board the Solar Dynamics Observatory (SDO). For a more comprehensive discussion of observations of SADs, readers are referred to our recent paper, Innes et al. (2014). Interestingly, the uniform resistivity simulation appears to be in better agreement with observations.

\section{Simulation}

In this section, we introduce the setup of our MHD simulation for studies of SADs, the simulation results, and examine their consistency with observations.

\subsection{Simulation setup}

Our numerical model solves the following normalized three-dimensional MHD equations 


$$
\begin{gathered}
\partial_{t} \rho=-\nabla \cdot(\rho \mathbf{v}), \\
\partial_{t}(\rho \mathbf{v})=-\nabla \cdot(\rho \mathbf{v} \mathbf{v})-\nabla p+\mathbf{J} \times \mathbf{B}+\mu \nabla^{2}(\rho \mathbf{v}), \\
\partial_{t} p=-\nabla \cdot(p \mathbf{v})-(\gamma-1) p \nabla \cdot \mathbf{v}+(\gamma-1) \eta J^{2}, \\
\partial_{t} \boldsymbol{B}=-\nabla \times(-\mathbf{v} \times \boldsymbol{B}+\eta \mathbf{J}) .
\end{gathered}
$$

Here $\rho$ is the plasma density, $\mathbf{v}$ is the plasmas velocity, $\mathbf{B}$ is the magnetic field, $\mathbf{J}=\nabla \times \mathbf{B}$ is the electric current density, $p$ is the plasma thermal pressure, $\mu$ is the plasma viscosity, and $\eta$ is the plasma resistivity. Our model includes ohmic heating, but exclude viscous heating, heat conduction and radiation cooling. Gravity is not included.

We consider a triply periodic system in the domain $-L_{x} \leq x \leq L_{x},-L_{y} \leq y \leq L_{y}$, $-L_{z} \leq z \leq L_{z}$. However, simulations are carried out in the region $0 \leq x \leq L_{x}$ and $0 \leq z \leq L_{z}$, and solutions in the remaining part of the domain are inferred by symmetry. The initial magnetic field is the Harris double current layer (TanDokoro \& Fujimoto 2005) defined as:

$$
B_{x}=\left\{\begin{array}{cc}
B_{0} \tanh (z / a) & |z| \leq L_{z} / 2 \\
-B_{0} \tanh \left(\left(z-L_{z}\right) / a\right) & z>L_{z} / 2
\end{array},\right.
$$

where $a$ is the Harris current sheet width, and $B_{0}$ is the asymptotic magnetic field strength in the upstream region. The plasma thermal pressure is calculated by the force balance condition:

$$
p+\frac{B_{x}^{2}}{2}=\frac{B_{0}^{2}}{2}
$$

and the density profile is determined by the ideal gas law $p=2 \rho T_{0}$, where $T_{0}$ is the constant initial temperature and the factor 2 is due to contributions from both electrons and ions. In normalized units, we set $T_{0}=0.125, B_{0}=1, a=1$, and $\mu=5 \times 10^{-4}$. The density $\rho=1$, the plasma beta $\beta \equiv 2 p / B_{0}^{2}=0.5$, and the Alfven speed $V_{A}=1$ in the asymptotic region. 
The initial density profile is seeded with a random noise of magnitude $3 \times 10^{-2}$ to facilitate the instability. The dimensions of the simulation box are $L_{x}=150, L_{y}=5, L_{z}=40$, with grids $n_{x}=800, n_{y}=150, n_{z}=400$, respectively. The $+x$ direction is interpreted as the sunward direction. With this basic setting, we have carried out simulations with uniform, as well as with spatially localized anomalous resistivity.

\subsection{Uniform resistivity run}

In the first run, we use a uniform resistivity $\eta=3 \times 10^{-3}$ for the whole simulation box. To start the reconnection, we add an initial perturbation to the magnetic field in the $x$ and $z$ directions:

$$
\begin{gathered}
\delta B_{x}=-0.025 B_{0} \frac{L_{x}}{L_{z}} \cos \left(\frac{\pi x}{L_{x}}\right) \sin \left(\frac{\pi z}{L_{z}}\right), \\
\delta B_{z}=0.025 B_{0} \sin \left(\frac{\pi x}{L_{x}}\right) \cos \left(\frac{\pi z}{L_{z}}\right) .
\end{gathered}
$$

Subsequently, magnetic reconnection occurs along an extended Sweet-Parker current sheet. Reconnected magnetic field lines form magnetic arcades in the downstream region, where plasma ejected by the reconnection outflows accumulate and form a high-density region. An interface is seen to form between lower density reconnection outflows and the higher density plasma above the top of the arcades. Eventually, the interface becomes wavy, and the small wavy fluctuations develop into finger-like structures. Panel (a) of Figure 1 shows the lower part of the extended Sweet-Parker current sheet and the cusp region at the top of flare arcades, where the finger-like structures form. Panel (b) of Figure 1 shows a $2 \mathrm{D}$ slice of the density profile in the $x-y$ plane at $z=0.1$, panel (c) shows the temperature profile and panel $(\mathrm{d})$ shows the expected count rate $(\mathrm{DN} / \mathrm{s} / \mathrm{pixel})$ in the AIA $131 \AA$ channel calculated from the simulation data at the same plane. The emission count rate is calculated according to the formula $C R=\int n^{2} f(T) d l D N / s \cdot p i x e l$, where 
$f(T)$ is the AIA $131 \AA$ response function (Lemen et al. 2012), where $n$ is the electron number density, $T$ is the temperature and $d l$ is the line element along the line of sight. To use the response function, the plasma density and temperature have to be converted to dimensional units. Here the density is converted by assuming that unit density in simulation equals $10^{9} \mathrm{~cm}^{-3}$. The temperature is converted by assuming that the initial normalized Alfven speed $V_{A}=1$ in the lobe corresponds to $V_{A}=1000 \mathrm{~km} / \mathrm{s}$, which gives the initial temperature $T_{\text {real }}=\frac{m_{p} V_{A}^{2}}{k} T_{\text {code }}=\frac{1.67 \times 10^{-27} \mathrm{~kg} \times\left(10^{6} \mathrm{~m} / \mathrm{s}\right)^{2}}{1.38 \times 10^{-23} \mathrm{~m}^{2} \mathrm{kgs}^{-2} \mathrm{~K}^{-1}} \times 0.125 \simeq 1.5 \times 10^{7} \mathrm{~K}$ for our simulation. Likewise, the initial normalized magnetic field in the lobe $\left(B_{\text {code }}=1\right)$ corresponds to $B_{\text {real }} \simeq 14$ Gauss, which is a reasonable value for the coronal magnetic field.

The finger-like structures are caused by plasma instabilities in the exhaust region of a reconnecting current sheet. As can be seen from Figure 1, the instabilities take place at the interface between lighter reconnection outflows and denser plasma (piled-up density in front of reconnection outflows). Because the reconnection outflows push the relatively stationary plasma ahead, the deceleration existing between lighter and denser plasma plays a role that is equivalent to gravity in the Rayleigh-Taylor instability. Furthermore, the magnetic field lines in the arcade are highly bent in the downstream region (Figure 1), producing unfavorable curvature that makes the system potentially unstable to the ballooning instability (cf. Bhattacharjee et al. 1998). While we have not carried out a detailed linear stability analysis and leave this to future work, we propose that the finger-like SADs in the downstream region are results of the nonlinear evolution of instabilities of the Rayleigh-Taylor/ballooning type.

The typical speed of the SADs (speed of the tip motion) in this run is $\sim 0.05 V_{A}$, which is comparable to the observed value. The instabilities also induce transverse motion of SADs. Figure 2 shows the flow pattern on the $x-y$ plane, overplotted on the density profile shown in color. Downward moving tadpoles and upward moving spikes can be seen 
in Figure 2, as well as vortices. The plasma in the cusp region/supra-arcade fan is quite turbulent due to secondary instabilities. The observations of eddies co-existing with SADs have been reported by McKenzie (2013).

In observations, the clusters of SADs happen repeatedly over the course of the supra-arcade evolution. We found a similar behavior in our simulation. From the four

panels of Figure 1, we see that there are two clusters of finger-like structures, one at $x \simeq 115$ and the other at $x \simeq 130$. Throughout the whole simulation, there are four clusters of finger-like structures. Because the resistivity is uniform in space and constant over time in this run, our simulation suggests that the intermittent formation of finger-like SADs does not necessarily require intermittent, locally enhanced resistivity, but can be attributed to ideal instabilities in the downstream region.

\subsection{Anomalous resistivity run}

To test how the reconnection mechanism may affect the instabilities in the downstream region, we have carried out a second run with Petschek-type reconnection triggered by introducing a locally enhanced anomalous resistivity $\eta=\eta_{0} \exp \left(-x^{2}-z^{2}\right)$, with $\eta_{0}=3 \times 10^{-3}$. The Petschek-type reconnection soon creates a shock-like front propagating along the $+x$ direction, with the $B_{z}$ component and the plasma density $\rho$ piled up on the downstream side of the front. This propagating front later develops wavy structures, which subsequently grow and became elongated in the $x$ direction, as shown in Figure 3 . Panel (a) of Figure 3 shows a projected view of magnetic field lines, and a slice of the $B_{z}$ profile at $z=0.1$. Panel (b) of Figure 3 shows a 2D slice of the density profile on the $x-y$ plane at $z=0.1$. Panel (c) shows the temperature profile, and panel (d) shows the AIA $131 \AA$ emission count rate calculated from the simulation data on the same plane. 
The finger-like structures in Figure 3 consist of two parts. The "tadpoles" move in the $+x$ (sunward) direction and have low density, high temperature, and weak emission, and the "spikes" are fingers developing toward the $-x$ (anti-sunward) direction with higher density, lower temperature, and brighter emission. At a later stage, secondary instabilities (e.g. the Kelvin-Helmholtz instability) may happen at the interface between tadpoles and spikes and eventually cause mixing of tadpoles and spikes. Figure 4 shows the line of sight (z-direction) averaged emission count rate at four different times. The top panel shows tadpole-like structures at $t=120$. In the second and the third panels $(t=140$ and 160), the tadpoles get thinner as they descend further. Finally, in the fourth panel $(t=180)$, the tadpoles merge into the brighter surroundings.

Note that although a locally enhanced anomalous resistivity is employed in this run, the localized resistivity is uniform in the $y$ direction (along the current sheet layer) and constant over time. Therefore, the finger-like SADs are caused by instabilities and not a direct result of anomalous resistivity. Furthermore, the SADs in this run are qualitatively similar to the ones we obtained with the uniform resistivity. This suggests that the reconnection mechanism in the upstream region does not directly affect instabilities in the downstream region. Nevertheless, there are some differences regarding where and when the SADs take place and the visual propagation speed of SADs, which we will discuss in the next section.

\section{Summary and Conclusions}

The mechanism causing the formation of SADs has been an open question since their first discovery. Existing simulations of SADs depend on intermittently and locally induced reconnection events to reproduce finger-like SADs along current sheet layers. In addition, questions such as why the speeds of SADs are much slower than the Alfven speed and how 
the density depletion regions within SADs manage to balance the thermal pressure from the surrounding plasmas for a few minutes remain unsolved. In this paper, we describe simulations to test the idea that SADs might be the result of essentially ideal plasma instabilities in the downstream region of a reconnection site. We implement resistive MHD models with both uniform and spatially localized anomalous resistivity. Finger-like structures are generated in both runs with different dynamic behaviors. The comparisons between our simulations and observations of SADs can be summarized as follows:

(1) Appearance. The downward developing parts (tadpoles) of the finger-like structures are characterized by low density, high temperature, and low EUV emission count rate (shown in Figure 1 and Figure 3). The upward developing parts (spikes) are characterized by higher density, relatively low temperature and higher EUV emission count rate. The features of the downward developing parts in the simulations are very similar to the observational features of SADs, while the upward developing parts resemble bright spikes observed among SADs. The relatively higher temperature in the low-density plasma of tadpoles keeps them in approximate force balance with surrounding plasma and spikes. Therefore, our simulations are able to successfully reproduce the observational features of SADs and explain why elongated SADs structures can exist for a few minutes. In addition, both simulations and observations show that SADs get thinner as they penetrate deeper into the bright fans. Our simulations suggest that this behavior may be due to shear flow instabilities between upward-moving, high density plasma in spikes and downward-moving, low density plasma in tadpoles, which eventually result in the merger of tadpoles with the surrounding plasma.

(2) The timeline and the location. Observations show that after the initial eruption of a CME, bright material starts to accumulate above existing coronal loops. After a while, SADs start to show up as dark flows penetrating the bright material above the corona 
loops and bright spikes start to appear between the SADs. The whole event can last for a few hours, during which SADs occur repeatedly. The observations suggest the following scenario. After a CME eruption, a post-eruption reconnection site is formed; as magnetic reconnection proceeds, reconnection outflows develop in both the sunward and anti-sunward directions. The sunward moving outflows stack over the existing magnetic arcades in the corona and heat up the surrounding plasma. As a result, bright fans appear above the arcades. Later on, a cluster of SADs develop at the top of the bright fans due to instabilities and the low density jet plasma descends while the bright spikes move upward. In the following several hours, clusters of SADs occur repeatedly. The magnetic configuration and the timeline of events described here are in good agreement with the ones in the simulation with uniform resistivity.

(3) Dynamic characteristics. In most events, the SADs move sunward at speeds much lower than the Alfven speed (Savage \& McKenzie 2011), typically 15\% of the Alfvén speed. In the run with uniform resistivity, the instabilities occur at the top of the magnetic arcades where reconnection outflows have decelerated almost to a standstill. The velocity of SADs are seen in the simulations is approximately $5 \%$ of the Alfvén speed, which is in the range of the observed values. However, in the run with anomalous resistivity, the instabilities develops at the shock-like front which propagates sun-ward at the Alfvénic speed, which is not in good agreement with observations. From this perspective, the MHD model with uniform resistivity agrees better with observations than the one with anomalous resistivity.

Our results indicate that the exhaust region of magnetic reconnection can become unstable to Rayleigh-Taylor type instabilities, and the resulting finger-like structures exhibit features qualitatively similar to observations of SADs. Most importantly, we have shown that SADs can arise without reconnection being patchy, i.e. localized in either space or time (or both). In fact, the reconnection site in both runs of this study does not develop 
apparent 3D (i.e. $y$-dependent) structures, and reconnection continues at a constant rate throughout the simulations. Of course, in reality the reconnection site is likely to exhibit 3D effects, either due to the local onset of reconnection or 3D instabilities in the current sheet. It will be interesting to study how the 3D nature of the reconnection site affects the structures in the exhaust region. Our simulations can also account for the observed transverse motion of SADs (e.g. merging and splitting of SADs and vortices around SADs). We also note that in the run with uniform resistivity, SADs formed at the top of fan spikes as seen in recent AIA observations (Innes et al. 2014). The transverse motion of plasma may be due to shearing instabilities between tadpoles and spikes. This kind of motion could make the local region quite turbulent, as shown in Figure 2.

In conclusion, our results suggest that Rayleigh-Taylor type instabilities in the downstream region of a reconnecting current sheet provide a plausible mechanism for the formation of SADs. Between the two models of resistivity employed in our simulations, the uniform resistivity appears to agree better with observations. Rayleigh-Taylor type instabilities have also been observed in recent fully kinetic particle-in-cell simulations (Vapirev et al. 2013). Further study of the instabilities with different underlying models and detailed comparison with observations should shed new light on the nature of SADs, as well as what can be learned about the structure of the reconnection site from the appearance of SADs.

This work was supported by the Department of Energy, Grant No. DE-FG0207ER46372, under the auspice of the Center for Integrated Computation and Analysis of Reconnection and Turbulence (CICART), the National Science Foundation, Grant No. PHY-0215581 (PFC: Center for Magnetic Self-Organization in Laboratory and Astrophysical Plasmas), NASA Grant Nos. NNX09AJ86G and NNX10AC04G, and NSF Grant Nos. ATM-0802727, ATM-090315 and AGS-0962698. We acknowledge beneficial 
conversations with Dr. Nicholas Murphy. 


\section{REFERENCES}

Asai, A., Yokoyama, T., Shimojo, M., Shibata, K., 2004, ApJ, 605, L77-L80

Bhattacharjee, A and Ma, Z.-W. and Wang, Xiaogang, Geophysical research letters 25, 6 (1998), pp. 861-864.

Cassak, P. A., Drake, J. F., Gosling, J. T., et al. 2013 ApJ, 775, L14

Costa, A., Elaskar, S., Fernández, a C. A., \& Martínez, G. 2009, MNRAS, 400, L85

Cécere, e M., Schneiter, M., Costa, A., Elaskar, S., \& Maglione, S. 2012, ApJ, 759, 79

Daughton, W., Roytershteyn, V., Albright, B., Karimabadi, H., Yin, L., 2009, Physics of Plasmas, 16, 072117

Hara, H., Tsuneta, S., Lemen, J. R., Acton, L. W., \& McTiernan, J. M. 1992, PASJ, 44, L135

Innes, D. E., McKenzie, D. E., \& Wang, T. 2003, Sol. Phys., 217, 247

Innes, D. E., McKenzie, D. E., \& Wang, T. 2003, Sol. Phys., 217, 267

Innes, D. E., Guo, L.-J., Bhattacharjee, A., Huang, Y.-M., arXiv preprint arXiv:1401.4057 (2014).

Khan, J. I., Bain, H. M., \& Fletcher, L., 2007, A\&A, 475, 333-340

Lemen, J. R. et al., 2012, Solar Physics, 275, 17-40

Lin, J., Ko, Y.-K., Sui, L., Raymond, J. C., \& Vourlida, A. 2005, ApJ, 622:1251-1264

Linton, M. G., Longcope, D. W., 2006, Earth Planets Space, 61, 573-576

Linton, M. G., De Vore, C. R., Longcope, D. W., 2009, ApJ, 642:1177-1192 
Liu, R., Lee, J.,Wang, T., Stenborg,G.,Liu, C., \&Wang, H. 2010, ApJ Letter, 723, L28-L33

Maglione, L. S., Schneiter, E. M., Costa, A., \& Elaskar, S. 2011, A\&A, 527, L5

McKenzie, D. E. \& Hudson, H. S. 1999, ApJ, 519, L93

McKenzie, D. E. \& Savage, S. L. 2011, ApJ, 735, L6

McKenzie, D. E. 2000, Sol. Phys., 195, 381

McKenzie, D. E. 2011, Physics of Plasmas, 18, 111205

McKenzie, D. E. 2013, ApJ, 766:39

Savage,S. L., \& McKenzie, D. E., 2011, ApJ, 730:98

Savage,S. L., \& McKenzie, D. E., Reeves, K. K., 2012, ApJ, 747, L40

Scott, R. B., Longcope, D. W., \& McKenzie, D. E., 2013, ApJ, 776, 54

Sheeley, N. R., Jr., \& Wang, Y.-M. 2002, ApJ, 579, 874

TanDokoro, R., \& Fujimoto, M., 2005, J. Geophys. Res. Letter, 32, L23102

Vapirev, A. E., Lapenta, G., Divin, A., Markidis, S., Henri, P., Goldman, M., \& Newman, D., 2013, J. Geophys. Res., 118, 1435 
(a)

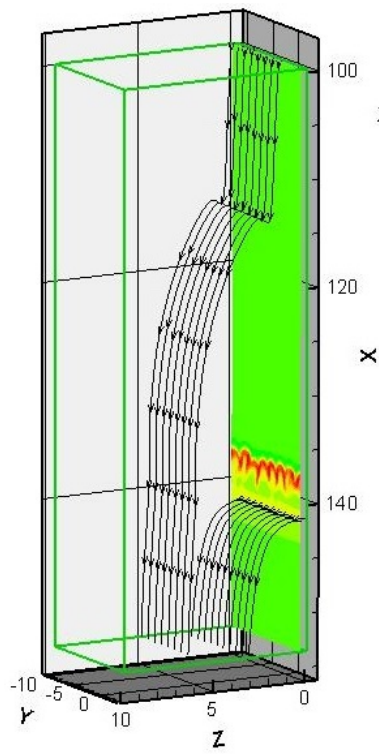

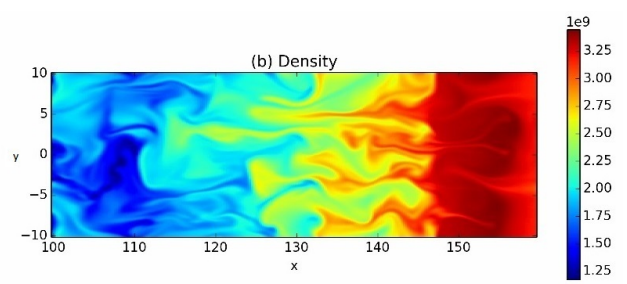
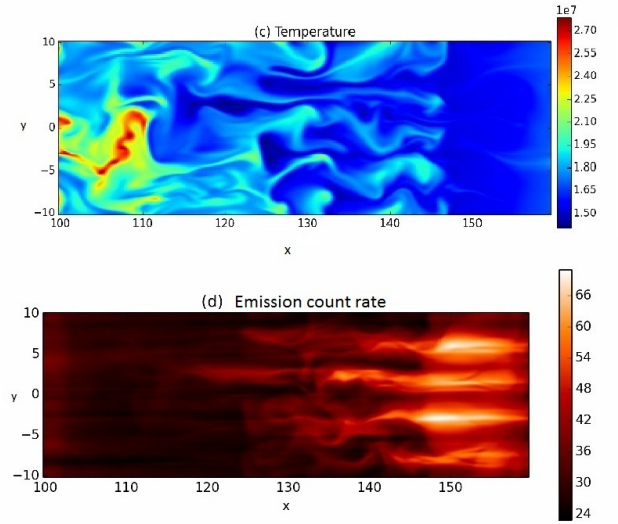

Fig. 1.- Panel (a) shows the magnetic field line configuration and a $x-y$ slice of $B_{z}$ profile at $z=0.1$ from MHD model with uniform resistivity, where the color codes represent the magnitude of the $B_{z}$ component (unit: Gauss). The z-axis in panel (a) is stretched by two times for a better projection. Panel (b) shows the density profile (unit : $/ \mathrm{cm}^{3}$ ), panel (c) shows the temperature profile (unit: Kelvin), and panel (d) shows the synthetic AIA $131 \AA$ emission count rate on the same $x-y$ plane.

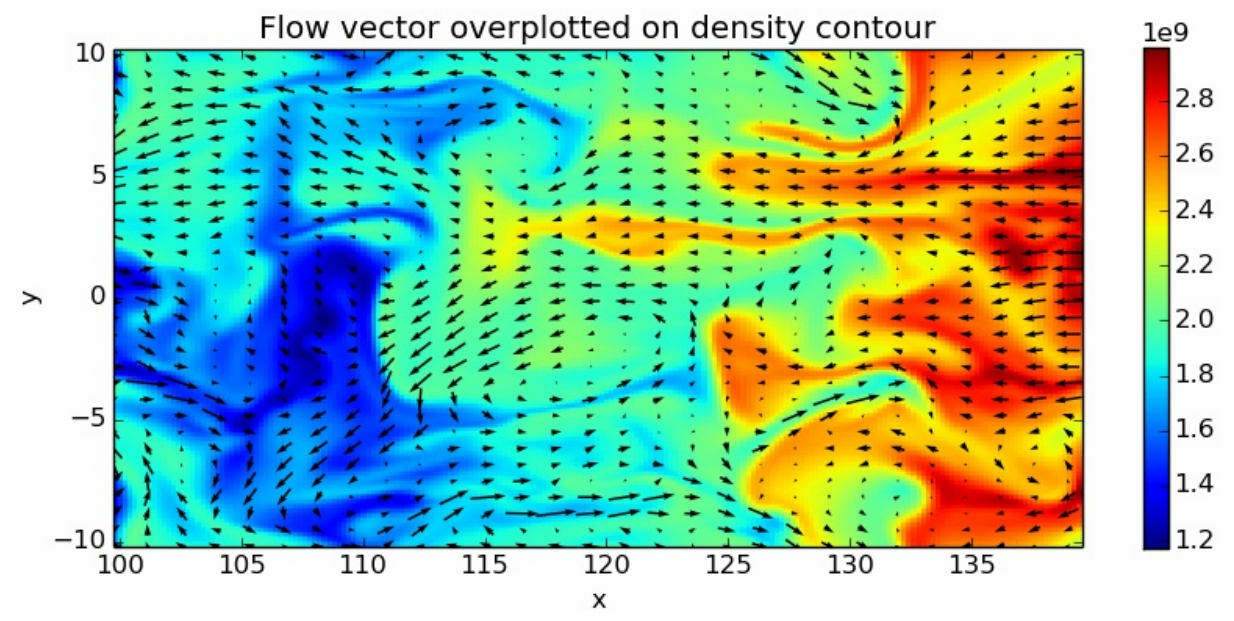

Fig. 2.- Flow pattern overplotted on a frame of the density profile. 

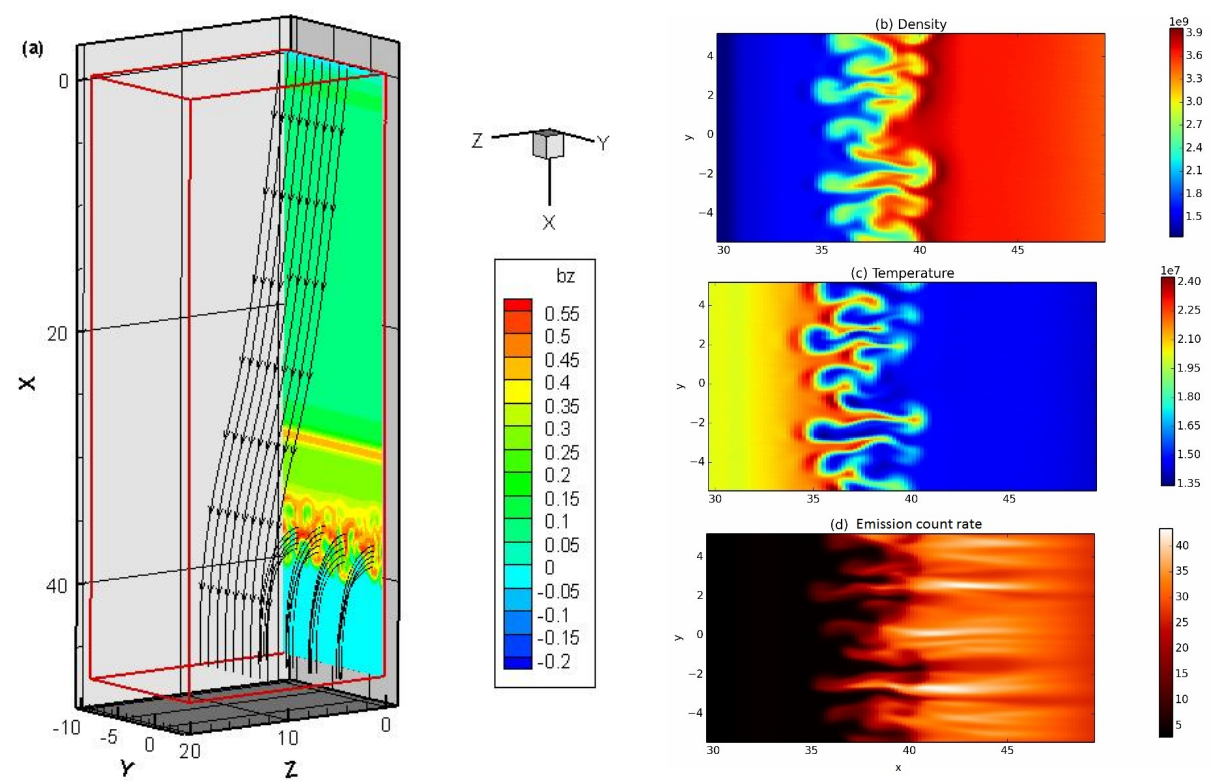

Fig. 3. - Panel (a) shows the magnetic field line configuration and a $x-y$ slice of $B_{z}$ profile for MHD model with anomalous resistivity. The $2 \mathrm{D} x-y$ slice is placed at $z=0.1$, where the color codes represent the magnitude of the $B_{z}$ component (unit: Gauss). Panel (b) shows the density profile (unit: $/ \mathrm{cm}^{3}$ ), panel (c) shows the temperature profile (unit: Kelvin), and panel (d) shows the synthetic AIA $131 \AA$ emission count rate on the same $x-y$ plane.
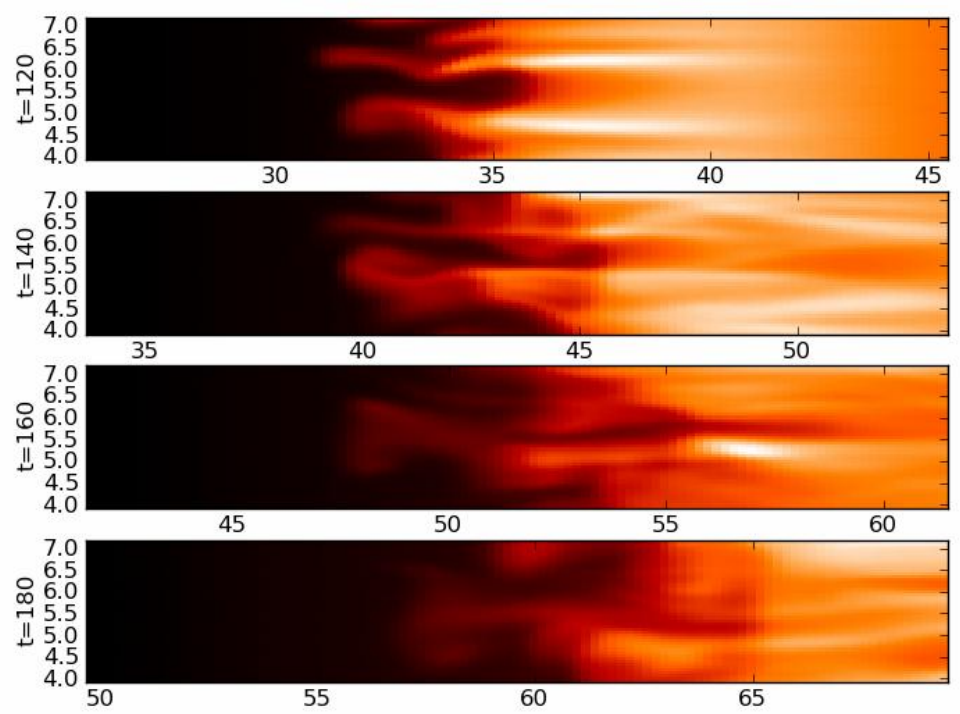
Fig. 4.- Line-of-sight averaged emission count rate featuring one SAD event developing along the $x$-direction at four different times. 